\title{
The effect of non-linear inertia on dynamic response of asymmetric multi-story buildings
}

\author{
Hassan Rezazadeh ${ }^{1}$, Fereidoun Amini² \\ School of Civil Engineering, Iran University of Science and Technology, Tehran, Iran \\ ${ }^{1}$ Corresponding author \\ E-mail: ${ }^{1}$ hrezazadeh@civileng.iust.ac.ir, ${ }^{2}$ famini@iust.ac.ir \\ Received 28 March 2018; accepted 4 April 2018 \\ DOI https://doi.org/10.21595/vp.2018.19856
}

Check for updates

\begin{abstract}
The goal of this paper is to inspect the influence of non-linear inertia on dynamic response of multi-story asymmetric buildings. In this study unlike conventional linear approach, the non-linear inertial terms are considered in the equations of motion. For considering non-linear inertia, the motion equations are derived in local rotary coordinates system. Also, the stiffness and damping parameters are defined in the rotary coordinates system. In the novel approach which is proposed in this paper, the motion equations are different with conventional linear approach. Numerical examples are presented to compare the response of the proposed model and conventional linear model. The modeled building response is derived under harmonic excitation. The results show that if non-linear inertia is considered, the dynamic response of asymmetric multi-story buildings may become different with conventional linear approach.
\end{abstract}

Keywords: non-linear inertia, asymmetric buildings, harmonic excitation.

\section{Introduction}

Torsional motions in asymmetric buildings and can cause severe damages [1]. So, it is important to carefully investigate the behavior of asymmetric buildings under dynamic loads. Many researchers have studied the effects of non-linear inertia on behavior of mechanical systems. Mayet and Ulbrich studied the non-linear detuning of centrifugal pendulum vibration absorber [2]. Mamandi et al. studied the non-linear behavior of an inclined beam subjected to a moving load [3]. In the field of structural engineering little attention has been paid to non-linear inertia and a few number of researchers have studied the effect of non-linear inertia on dynamic response of structural systems. Amin Afshar and Aghaei Pour studied the inertia non-linearity in irregular-plan isolated structures under seismic excitations [4]. Amini and Amin Afshar studied the effect of non-linear inertia on dynamic response of single story asymmetric building [5].

The effect of non-linear inertia in dynamic response of multi-story asymmetric buildings has not been studied enough. In this study non-linear inertia is considered in equations of motion and for considering non-linear inertia the equations of motion in each story is defined in local rotary coordinates system.

\section{Non-linear differential equations of motion}

As seen in Fig. 1(a), a single story building is subjected to ground excitation in excitation in $X$ and $Y$ directions. The floor mass center is denoted by C.M. and C.R. represents the center of stiffness of the story. Based on Amini and Amin Afshar approach, the non-linear equations of motion in local rotary $x y z$ coordinates system can be expressed as (see Fig. 1(b)) [5]:

$$
\begin{aligned}
& m \ddot{u}_{x}+C_{x} \dot{u}_{x}+K_{x} u_{x}=-m\left(-2 \dot{u}_{y} \dot{\theta}-u_{y} \ddot{\theta}-u_{x} \dot{\theta}^{2}+\ddot{u}_{g X} \cos \theta+\ddot{u}_{g Y} \sin \theta\right), \\
& m \ddot{u}_{y}+C_{y} \dot{u}_{y}+K_{y} u_{y}+e_{x} K_{y} \theta=-m\left(2 \dot{u}_{x} \dot{\theta}+u_{x} \ddot{\theta}-u_{y} \dot{\theta}^{2}-\ddot{u}_{g X} \sin \theta+\ddot{u}_{g Y} \cos \theta\right), \\
& m r^{2} \ddot{\theta}+C_{\theta} \dot{\theta}+\left(K_{\theta R}+K_{y} e_{x}^{2}\right) \theta+e_{x} K_{y} u_{y}=0 .
\end{aligned}
$$

In Eqs. (1)-(3), $e_{x}$ is the distance between C.M. and C.R. It is assumed that C.R. is located on $X$ axis. The value of $e_{x}$ can be calculated by: 
$e_{x}=\frac{\sum_{j=1}^{N} x_{j} k_{y j}}{\sum_{j=1}^{N} k_{y j}}$,

where $k_{y j}$ represents the stiffness of the $j$ th element resisting in $Y$ direction. In Eqs. (1)-(3), $m$ denotes the total mass of the floor and $r$ is the floor radius of gyration about the mass center, $\ddot{u}_{g X}$ and $\ddot{u}_{g Y}$ are the ground accelerations in $X$ and $Y$ directions, $u_{x}$ and $u_{y}$ are displacements of the floor mass center in the $x$ and $y$ directions and $\theta$ is the rotation of the floor about the $z$ axis. Also, $K_{x}$ and $K_{y}$ are total stiffness of the story in the $x$ and $y$ directions. The parameter $K_{\theta R}$ is the torsional stiffness of the story about C.R. and can be calculated by:

$K_{\theta R}=\sum_{j=1}^{M} K_{x j} y_{j}^{2}+\sum_{j=1}^{N} K_{y j}\left(x_{j}-e_{x}\right)^{2}$,

where $K_{x j}$ represents the stiffness of the $j$ th resisting element in $X$ direction. Moreover $C_{x}, C_{y}$ and $C_{\theta}$ are damping coefficients [5].

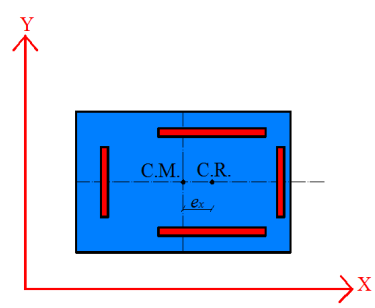

a)

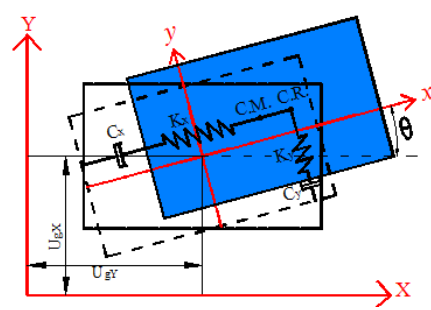

b)

Fig. 1. a) The plan of asymmetric building, b) global $(X Y Z)$ and local $(x y z)$ coordinates systems; - structure elements [4]

The motion equations of the multi-story building will be derived according to Eqs. (1)-(3). The equations of motion of the $i$ th story will be derived in the local rotary $x_{i} y_{i} z_{i}$ system of coordinates. As seen in Fig. 2 the $x_{i} y_{i} z_{i}$ coordinates system is located on the base of the building and rotates by an angle $\theta_{i}$. It should be noted that in the conventional linear approach the motion equations are derived in fixed $X Y Z$ system of coordinates. The motion equations of the $i$ th story can be expressed as [6]:

$M_{i} a_{i}^{i}+C_{i}^{i} \dot{\bar{U}}_{i}^{i}+K_{i}^{i} \bar{U}_{i}^{i}-C_{i+1}^{i} \dot{\bar{U}}_{i+1}^{i}-K_{i+1}^{i} \bar{U}_{i+1}^{i}=0$,

where $M_{i} a_{i}^{i}$ denotes the inertial forces, which is exerted to the $i$ th floor. Also the term $K_{i}^{i} \bar{U}_{i}^{i}$ is the force of resisting elements in $i-1$ th story which is applied to the $i$ th floor and the term $K_{i+1}^{i} \bar{U}_{i+1}^{i}$ is the force of resisting elements in $i+1$ th story which is exerted to the $i$ th floor. Also, the terms $C_{i}^{i} \dot{\bar{U}}_{i}^{i}$ and $C_{i+1}^{i} \dot{\bar{U}}_{i+1}^{i}$ denote the damping forces. In Eq. (6), $M_{i}$ denotes the mass matrix of the $i$ th floor. The matrix $M_{i}$ can be expressed as:

$M_{i}=\left[\begin{array}{ccc}m_{i} & 0 & 0 \\ 0 & m_{i} r_{i}^{2} & 0 \\ 0 & 0 & m_{i}\end{array}\right]$,

where $m_{i}$ is the mass of the $i$ th floor and $r_{i}$ is the $i$ th floor radius of gyration about the mass center. In Eq. (6), $a_{i}^{i}$ represents the total acceleration of the $i$ th floor center of mass in the local $x_{i} y_{i} z_{i}$ coordinates system. The vector $a_{i}^{i}$ can be calculated by [6]: 
$a_{i}^{i}=\left\{\begin{array}{l}a_{x i}^{i} \\ a_{\theta i} \\ a_{y i}^{i}\end{array}\right\}=\left\{\begin{array}{l}\ddot{u}_{x i}^{i} \\ \ddot{\theta}_{i} \\ \ddot{u}_{y i}^{i}\end{array}\right\}+\left\{\begin{array}{c}-2 \dot{u}_{y i}^{i} \dot{\theta}_{i}-u_{y i}^{i} \ddot{\theta}_{i}-u_{x i}^{i} \dot{\theta}_{i}^{2} \\ 0 \\ 2 \dot{u}_{x i}^{i} \dot{\theta}_{i}+u_{x i}^{i} \ddot{\theta}_{i}-u_{y i}^{i} \dot{\theta}_{i}^{2}\end{array}\right\}+\left\{\begin{array}{c}\ddot{U}_{X g} \cos \theta_{i}+\ddot{U}_{Y g} \sin \theta_{i} \\ 0 \\ -\ddot{U}_{X g} \sin \theta_{i}+\ddot{U}_{Y g} \cos \theta_{i}\end{array}\right\}$,

where $u_{x i}^{i}$ and $u_{y i}^{i}$ are displacements of the $i$ th floor mass center in $x_{i}$ and $y_{i}$ directions (see Fig. 2), $\ddot{U}_{X g}$ and $\ddot{U}_{Y g}$ are the ground translational accelerations. Moreover in Eq. (6), $K_{i}^{i}$ is the stiffness matrix of the $i$ th story. The matrix $K_{i}^{i}$ is defined in the directions of $x_{i} y_{i} z_{i}$ coordinates system. The matrix $K_{i}^{i}$ can be expressed as:

$K_{i}^{i}=\left[\begin{array}{ccc}K_{x i}^{i} & 0 & 0 \\ 0 & K_{\theta i} & K_{y i}^{i} e_{x i} \\ 0 & K_{y i}^{i} e_{x i} & K_{y i}^{i}\end{array}\right]$

where $K_{x i}^{i}$ and $K_{y i}^{i}$ are the $i$ th story stiffness in the $x_{i}$ and $y_{i}$ directions. The parameters $K_{x i}^{i}$ and $K_{y i}^{i}$ are defined in the rotary $x_{i} y_{i} z_{i}$ coordinates system. The parameter $K_{\theta i}^{i}$ is the torsional stiffness of the $i$ th story about the $i$ th story center of stiffness. Also, $e_{x i}$ is the distance between the mass center and the center of the stiffness of the $i$ th story. In Eq. (6), $C_{i}^{i}$ is the damping matrix of the $i$ th story. The matrix $C_{i}^{i}$ can be expressed as:

$C_{i}^{i}=\left[\begin{array}{ccc}C_{x i}^{i} & 0 & 0 \\ 0 & C_{\theta i} & C_{y i}^{i} e_{x i} \\ 0 & C_{y i}^{i} e_{x i} & C_{y i}^{i}\end{array}\right]$

where $C_{x i}^{i}, C_{y i}^{i}$ and $C_{\theta i}$ are the $i$ th story damping coefficients. These coefficients are defined in $x_{i} y_{i} z_{i}$ coordinates system. If $U_{X i}$ and $U_{Y i}$ represent the displacements of the $i$ th floor center of mass in the $X$ and $Y$ direction (see Fig. 2), the relationship between $\left[U_{X i}, \theta_{i}, U_{Y i}\right]^{T}$ and $\left[u_{x i}, \theta_{i}, u_{y i}\right]^{T}$ vectors can be expressed as:

$U_{i}^{i}=\left\{\begin{array}{c}u_{x i}^{i} \\ \theta_{i} \\ u_{y i}^{i}\end{array}\right\}=\left[\begin{array}{ccc}\cos \theta_{i} & 0 & \sin \theta_{i} \\ 0 & 1 & 0 \\ -\sin \theta_{i} & 0 & \cos \theta_{i}\end{array}\right]\left\{\begin{array}{c}U_{X i} \\ \theta_{i} \\ U_{Y i}\end{array}\right\}=Q^{i} U^{i}$,

where $Q^{i}$ is the rotation matrix about the $z_{i}$ axis by an angle $\theta_{i}$. Also, the vector $U_{i+1}^{i+1}$ is defined by:

$U_{i+1}^{i+1}=\left[u_{x_{i+1}}^{i+1}, \theta_{i+1}, u_{y_{i+1}}^{i+1}\right]^{T}$

Moreover in Eq. (6), $\bar{U}_{i}^{i}$ and $\bar{U}_{i+1}^{i}$ are defined by:

$\bar{U}_{i}^{i}=U_{i}^{i}-U_{i-1}^{i}$,

$\bar{U}_{i+1}^{i}=U_{i+1}^{i}-U_{i}^{i}$,

where the vector $U_{i-1}^{i}$ denotes the $i-1$ th floor displacement. Also $U_{i+1}^{i}$ is the $i+1$ th floor displacement vector. The vectors $U_{i-1}^{i}$ and $U_{i+1}^{i}$, are defined in the rotary $x_{i} y_{i} z_{i}$ coordinates system. The relationship between $U_{i+1}^{i}$ and $U_{i+1}^{i+1}$ can be expressed as: 
$U_{i+1}^{i}=\left\{\begin{array}{c}u_{x i+1}^{i} \\ \theta_{i+1} \\ u_{y i+1}^{i}\end{array}\right\}=\left[\begin{array}{ccc}\cos \bar{\theta}_{i+1} & 0 & -\sin \bar{\theta}_{i+1} \\ 0 & 1 & 0 \\ \sin \bar{\theta}_{i+1} & 0 & \cos \bar{\theta}_{i+1}\end{array}\right]\left\{\begin{array}{c}u_{x i+1}^{i+1} \\ \theta_{i+1} \\ u_{y i+1}^{i+1}\end{array}\right\}=\left(\bar{Q}_{i}^{i+1}\right)^{T} u_{i+1}^{i+1}$,

where $\bar{\theta}_{i+1}$ is defined by $\bar{\theta}_{i+1}=\theta_{i+1}-\theta_{i}$.

Now in Eq. (6), the matrix $K_{i+1}^{i}$ will be derived. The vector $F_{i+1}^{i+1}$ is defined by:

$F_{i+1}^{i+1}=K_{i+1}^{i+1} \bar{U}_{i+1}^{i}$

As it was mentioned before, the vector $F_{i+1}^{i+1}$ denotes the force of the resisting elements in $i+1$ th story which is applied to the $i+1$ th floor. Vector $F_{i+1}^{i+1}$ is defined in $x_{i+1} y_{i+1} z_{i+1}$ coordinates system. It is obvious that the reaction of the vector $F_{i+1}^{i+1}$ is applied to the $i$ th floor. To define the vector $F_{i+1}^{i+1}$ in $x_{i} y_{i} z_{i}$ system of coordinates, it can be rotated about $z_{i}$ axis by angle $-\bar{\theta}_{i+1}$. So, following equation can be written:

$F_{i+1}^{i}=-\left(\bar{Q}_{i}^{i+1}\right)^{T} F_{i+1}^{i+1}$,

where vector $F_{i+1}^{i}$ is the $i+1$ th story resisting elements force which is applied to the $i$ th floor. Vector $F_{i+1}^{i}$ is defined in $x_{i} y_{i} z_{i}$ coordinates system. As it was mentioned earlier, in Eq. (6) vector $F_{i+1}^{i}$ is defined by:

$F_{i+1}^{i}=-K_{i+1}^{i} \bar{U}_{i+1}^{i}$.

According to Eqs. (17)-(19) the term $-K_{i+1}^{i} \bar{U}_{i+1}^{i}$ can be expressed as:

$$
\begin{aligned}
& F_{i+1}^{i}=-\left(\bar{Q}_{i}^{i+1}\right)^{T} F_{i+1}^{i+1}=-\left(\bar{Q}_{i}^{i+1}\right)^{T} K_{i+1}^{i+1}\left(\bar{U}_{i+1}^{i+1}\right)=-\left(\bar{Q}_{i}^{i+1}\right)^{T} K_{i+1}^{i+1}\left(\bar{Q}_{i}^{i+1}\left(\bar{U}_{i+1}^{i}\right)\right) \\
& \quad=-\left(\left(\bar{Q}_{i}^{i+1}\right)^{T} K_{i+1}^{i+1}\left(\bar{Q}_{i}^{i+1}\right)\right) \bar{U}_{i+1}^{i},
\end{aligned}
$$

and so $K_{i+1}^{i}$ can be expressed as:

$K_{i+1}^{i}=\left(\bar{Q}_{i}^{i+1}\right)^{T} K_{i+1}^{i+1}\left(\bar{Q}_{i}^{i+1}\right)$.

Also, similar method can be used to derive $C_{i+1}^{i}$ and it can be expressed as:

$C_{i+1}^{i}=\left(\bar{Q}_{i}^{i+1}\right)^{T} C_{i+1}^{i+1}\left(\bar{Q}_{i}^{i+1}\right)$,

where $C_{i+1}^{i+1}$ is the $i+1$ th floor damping matrix which is defined in $x_{i+1} y_{i+1} z_{i+1}$ coordinates system.

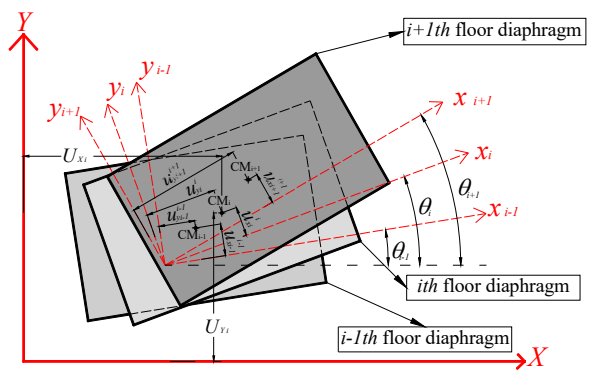

Fig. 2. Local rotary $x_{i} y_{i} z_{i}$ coordinates system; $C M_{i}$ denotes the $i$ th floor center of mass 


\section{Numerical studies}

Here, for comparing the response of proposed model and conventional linear model a five story building model is considered. The modeled building properties are listed in Table 1. It should be noted that the modeled structures is a scaled buildings. The damping ratio of the modeled building is selected to be $0.5 \%$. Moreover, the natural frequencies of the first three modes are listed in Table 2. The ground harmonic excitation in $X$ and $Y$ directions is selected to be:

$\ddot{U}_{g X}=A \sin (\omega t) \cos (\beta), \quad \ddot{U}_{g Y}=A \sin (\omega t) \sin (\beta)$,

where the parameter $A$ denoted the amplitude of the excitation and $\beta$ is the excitation arrival angle with respect to $X$ direction.

Table 1. Modeled building properties

\begin{tabular}{|c|c|c|c|c|c|}
\hline Story No. & Mass $\left(\mathrm{N} /\left(\mathrm{cm} / \mathrm{s}^{2}\right)\right)$ & $K_{x}$ and $K_{y}(\mathrm{~N} / \mathrm{cm})$ & $K_{\theta R}(\mathrm{~N} . \mathrm{cm})$ & $e_{x}(\mathrm{~cm})$ & $R(\mathrm{~cm})$ \\
\hline 1 & 0.1 & 142.1 & 32095 & 9.67 & 20 \\
\hline 2 & 0.1 & 142.1 & 32095 & 9.67 & 20 \\
\hline 3 & 0.1 & 142.1 & 32095 & 9.67 & 20 \\
\hline 4 & 0.1 & 142.1 & 32095 & 9.67 & 20 \\
\hline 5 & 0.1 & 142.1 & 32095 & 9.67 & 20 \\
\hline
\end{tabular}

Table 2. Modeled building natural frequencies in first thee modes

\begin{tabular}{|c|c|c|}
\hline$\omega_{1}(\mathrm{rad} / \mathrm{s})$ & $\omega_{2}(\mathrm{rad} / \mathrm{s})$ & $\omega_{3}(\mathrm{rad} / \mathrm{s})$ \\
\hline 5.39 & 10.73 & 12.29 \\
\hline
\end{tabular}

The response of top floor mass center is shown in Fig. 3. It is seen that the response of linear and non-linear models is quite different. The response of top floor mass center in linear model and $X$ direction is zero. But in non-linear model the top story mass center oscillates in $X$ direction. In non-linear model, after initial steps of excitation the amplitude of the response in $X$ direction increases. Moreover, in non-linear model the amplitude of the response in $Y$ direction reaches a constant value after initial oscillations. It is seen that when the amplitude of excitation is increased, the difference between linear and non-linear model responses become more.

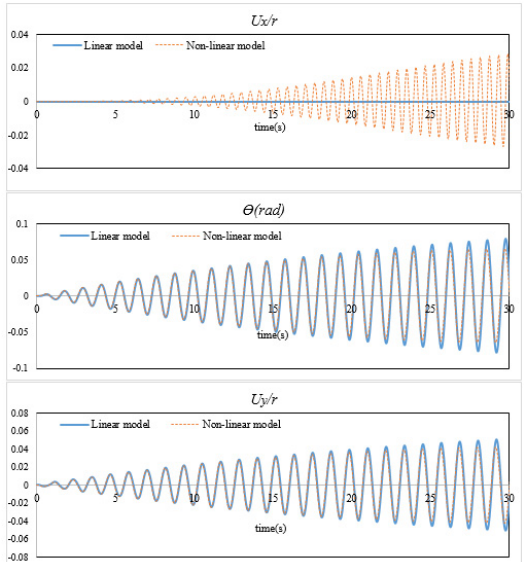

a)

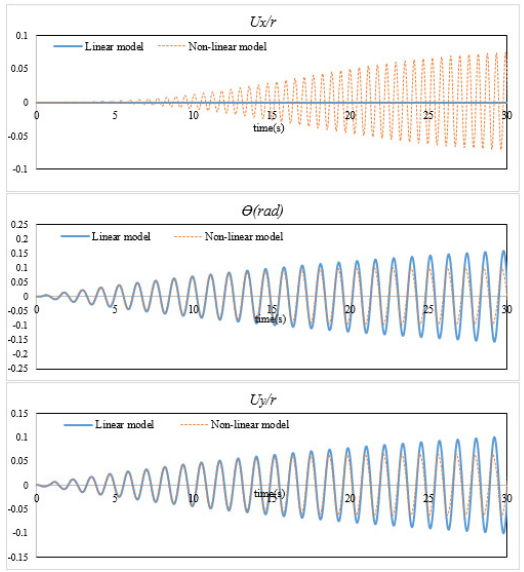

b)

Fig. 3. Top story response of the modeled building: a) $A=0.005, \beta=90^{\circ}$ and $\omega=\omega_{1}$, b) $A=0.01, \beta=90^{\circ}$ and $\omega=\omega_{1}$

In Fig. 4 the response of the top floor mass center is shown. In this figure the parameter $\beta$ is selected to be near to zero. In Fig. 4(a), the response of the both linear and non-linear models are 
identical in $X$ direction and the difference between these two models can be ignored. In the response of $Y$ direction and $\theta$, linear and non-linear models have the same response in initial steps of excitation. But after initial steps of oscillation the response of $Y$ direction and $\theta$ increases.

As seen in Fig. 4 when the excitation amplitude is increased, the difference between linear and non-linear models becomes more. In Fig. 4(b) when the amplitude of the response in $X$ direction reaches a certain value; the amplitude suddenly decreases in $X$ direction and again increases. Moreover, after initial steps of excitation the response of $Y$ direction $\theta$ increases. When the energy amount in the dominant mode of $X$ direction reaches a certain value, this mode is saturated, and the energy is transferred to other modes.

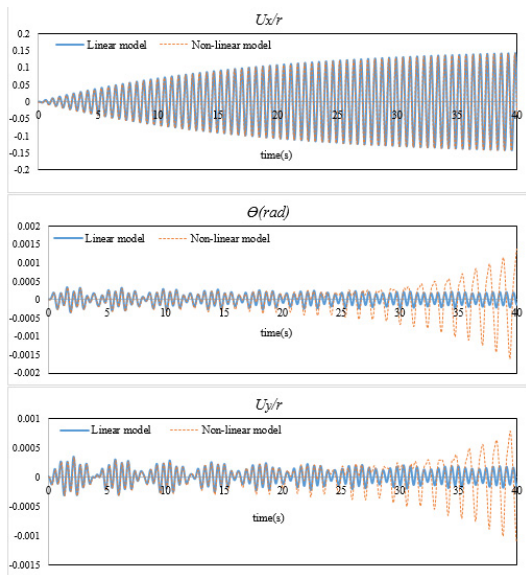

a)

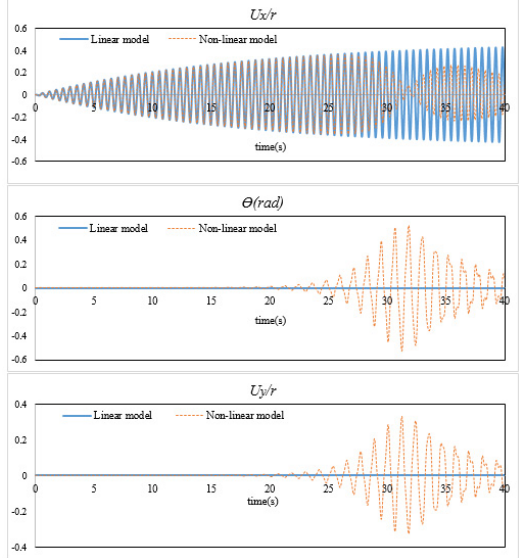

b)

Fig. 4. Top story response of the modeled building: a) $A=0.01, \beta=0.05 \mathrm{rad}$ and $\omega=\omega_{2}$, b) $A=0.03, \beta=0.05 \mathrm{rad}$ and $\omega=\omega_{2}$

\section{Conclusions}

In this study unlike conventional linear approach, the non-linear inertia was considered in equations of motion of multi-story asymmetric building. For considering non-linear inertia, the equations of motion were derived in local rotary coordinates system. It was seen that the conventional linear approach is weak to model the behavior of asymmetric buildings and considering non-linear inertia leads to different response. In the modeled structure if the amplitude of the excitation is increased the difference between linear and proposed non-linear models become more. Also, in some cases energy transfer between the modes is observed.

\section{References}

[1] Amin Afshar M., Amini F. Non-linear dynamics of asymmetric structures under 2:2:1 resonance. International Journal of Non-Linear Mechanics, Vol. 47, 2012, p. 823-835.

[2] Mayet J., Ulbrich H. First-order optimal linear and nonlinear detuning of centrifugal pendulum vibration absorbers. Journal of Sound and Vibration, Vol. 335, 2015, p. 34-54.

[3] Mamandi A., Kargarnovin M. H., Younesian D. Nonlinear dynamics of an inclined beam subjected to a moving load. Nonlinear Dynamics, Vol. 60, 2010, p. 277-293.

[4] Amin Afshar M., Aghaei Pour S. On inertia nonlinearity in irregular-plan isolated structures under seismic excitations. Journal of Sound and Vibration, Vol. 363, 2016, p. 495-516.

[5] Amini F., Amin Afshar M. Saturation in asymmetric structures under internal resonance. Acta Mechanica, Vol. 221, 2011, p. 353-368.

[6] Amini F., Rezazadeh H., Amin Afshar M. Adaptive control of rotationally non-linear asymmetric structures under seismic loads. Structural Engineering and Mechanics, Vol. 65, Issue 6, 2018, p. 721-730. 